Copyright (C) 1990, by the author(s).

All rights reserved.

Permission to make digital or hard copies of all or part of this work for personal or classroom use is granted without fee provided that copies are not made or distributed for profit or commercial advantage and that copies bear this notice and the full citation on the first page. To copy otherwise, to republish, to post on servers or to redistribute to lists, requires prior specific permission. 


\section{SIMULTANEOUS POTENTIAL AND CIRCUIT SOLUTION FOR BOUNDED PLASMA PARTICLE SIMULATION CODES}

by

John P. Verboncoeur, M. Virginia Alves, and V. Vahedi

Memorandum No. UCB/ERL M90/67

7 August 1990 


\title{
SIMULTANEOUS POTENTIAL AND CIRCUIT SOLUTION FOR BOUNDED PLASMA PARTICLE SIMULATION CODES
}

\author{
by \\ John P. Verboncoeur, M. Virginia Alves, and V. Vahedi
}

Memorandum No. UCB/ERL M90/67

7 August 1990

\section{ELECTRONICS RESEARCH LABORATORY}

\author{
College of Engineering \\ University of California, Berkeley \\ 94720
}




\section{SIMULTANEOUS POTENTIAL AND CIRCUIT SOLUTION FOR BOUNDED PLASMA PARTICLE SIMULATION CODES}

by

John P. Verboncoeur, M. Virginia Alves, and V. Vahedi

Memorandum No. UCB/ERL M90/67

7 August 1990

\section{ELECTRONICS RESEARCH LABORATORY}

College of Engineering

University of California, Berkeley

94720 


\title{
SIMULTANEOUS POTENTIAL AND CIRCUIT SOLUTION FOR BOUNDED PLASMA PARTICLE SIMULATION CODES
}

\author{
John P. Verboncoeur, M. Virginia Alves ${ }^{\dagger}$, and V. Vahedi \\ Plasma Theory and Simulation Group \\ University of California, Berkeley, CA 94720
}

\begin{abstract}
A second-order accurate method for solving the combined potential and circuit equations in an electrostatic bounded plasma PIC simulation is presented. The boundary conditions include surface charge on the electrodes, which are connected to a series RLC circuit with driving terms $V(t)$ and $I(t)$. The solution is obtained for planar, cylindrical, and spherical electrodes. The result is a tridiagonal matrix which is readily solved using well-known methods. The method is implemented in the codes PDP1 (Plasma Device Planar 1D), PDC1 (Cylindrical), and PDS1 (Spherical).
\end{abstract}

\section{INTRODUCTION}

A comprehensive review of the considerations involved in bounded plasma particle simulation is presented by W. S. Lawson [1]. Lawson presents a method which is second-order accurate when $\Delta t^{2} / L C \ll 1$ and $R \Delta t / L \ll 1$, and is stable for $\Delta t^{2} / L C<2$ and $R \Delta t / L<2$. Here we improve on the accuracy, stability, and simultaneity of the solution for potentials in a bounded onedimensional plasma with external circuit and driving terms.

In [1] and [2] the boundary conditions are decoupled from the potential equation. A firstorder circuit solution is used when the inductance is zero. The scheme is self-consistent when $L$ is non-zero and the applied (driving) potential is small compared to the space-charge potential across the system. These conditions are violated for a large class of problems, including capacitively coupled RF discharges and plasma immersion ion implantation materials processing; therefore, a new method is desired.

Particle-in-Cell (PIC) methods weight particles to a spatial grid using a particle shape factor to obtain charge and current densities on the grid [2]. For example, the code PDP1 uses the linear weighting scheme shown in Figure 1. The field and circuit solution presented here is independent of the weighting scheme used; we assume that the charge density is given on the spatial grid.

$\dagger$ Permanent address Institute for Space Research (INPE), P.O. Box 515, S. J. dos Campos, SP, 12201, Brazil; supported in part by CAPES, Ministry of Education, Brazil. 

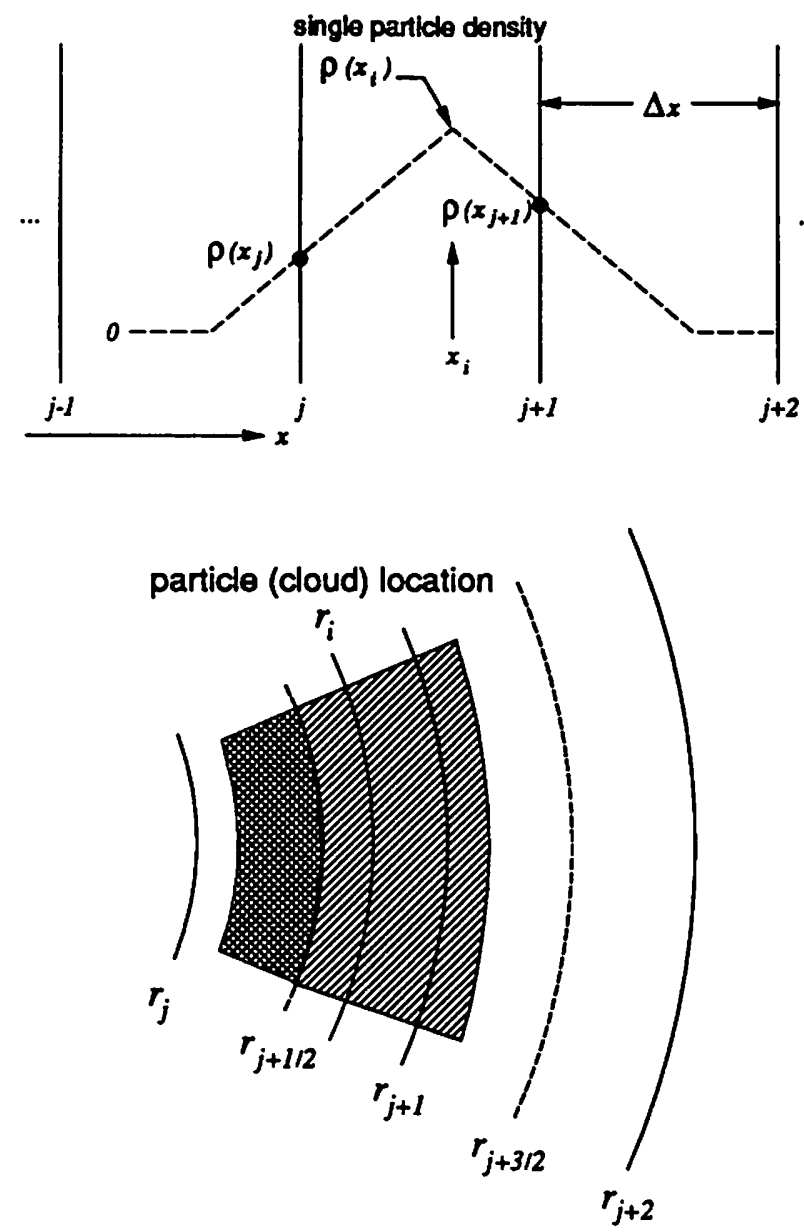

Figure 1. PDP1 PIC with linear weighting to the spatial grid. The subscript $i$ is the particle index, $j$ is ... the grid index. For particles in a cell adjacent to an electrode, the weighting must also account for the half width of the cell.

Particles of finite size, cylindrical shells in PDC1 and spherical shells in PDS1, are placed in a gridded system, and weighted to the grid to obtain $\rho\left(r_{j}\right)$ at the grid points. The particles are assumed to have uniform density, so the area of rings or the volume of shells can be used to weight the charges to the grid as shown in Figure 2. The particle of finite width $\Delta r$ is centered at $r_{i}$. The intersection between the finite particle and the grid cell determines the fraction of the particle charge assigned to each grid node $r_{j}$. This is cloud-in-cell weighting, versus the particle-in-cell weighting in [2, Figure 14-11a]. The fractions of the charge assigned to the grids are

$$
s_{j}=\frac{r_{j+1 / 2}^{2}-r_{i-1 / 2}^{2}}{r_{i+1 / 2}^{2}-r_{i-1 / 2}^{2}}
$$

and

$$
s_{j+1}=\frac{r_{i+1 / 2}^{2}-r_{j+1 / 2}^{2}}{r_{i+1 / 2}^{2}-r_{i-1 / 2}^{2}} .
$$


In the spherical model, the squared terms are replaced by cubic terms.

The charge density on the grid is used to solve the Poisson equation, $\nabla^{2} \Phi=-\rho / \varepsilon$, or the equivalent flux conserving potential equation obtained from Gauss' Law. Once the potentials are known, the electric field on the grid can be obtained from $E=-\nabla \Phi$. The force on the particles is obtained by interpolating $\mathbf{E}$ at each particle position using some weighting. The particle velocity is updated using the Lorentz force equation,

$$
\frac{d \mathbf{v}_{i}}{d t}=\frac{q_{i}}{m_{i}}\left[\mathrm{E}\left(x_{i}\right)+\mathbf{v}_{i} \times \mathbf{B}\right] .
$$

The particle positions are updated using

$$
\frac{d \mathrm{x}_{i}}{d t}=\mathrm{v}_{i}
$$

In cylindrical coordinates, Eq. (4) is

$$
\begin{gathered}
m \frac{d v_{r}}{d t}=F_{r}+\frac{m v_{\theta}^{2}}{r}, \\
m \frac{d\left(r v_{\theta}\right)}{d t}=r F_{\theta}, \text { and } \\
m \frac{d v_{z}}{d t}=F_{z} .
\end{gathered}
$$

Here $r, \theta$, and $z$ are the cylindrical coordinates and $v_{r}=d r / d t$ and $v_{\theta}=r d \theta / d t$. For the cylindrical model, $\mathrm{B}=B_{z} \hat{z}$ so $F_{r}=q\left(E_{r}+v_{\theta} B_{z}\right), F_{\theta}=-q v_{r} B_{z}$, and $F_{z}=0$. Equation (5) is finite differenced to obtain the sequence

$$
\begin{gathered}
v_{r}^{t+\Delta / 2}=v_{r}^{t-\Delta / 2}+\Delta t\left(\frac{\left(v_{\theta}^{t}\right)^{2}}{r^{t}}+\frac{F_{r}^{t}}{m}\right), \\
r^{t+\Delta t}=r^{t}+\Delta t v_{r}^{t+\Delta / 2} \text {, and } \\
v_{\theta}^{t+\Delta t}=\frac{r^{t} v_{\theta}^{t}}{r^{t+\Delta t}}+\frac{q B_{z}}{2 m r^{t+\Delta t}}\left[\left(r^{t}\right)^{2}-\left(r^{t+\Delta t}\right)^{2}\right] .
\end{gathered}
$$

The latter guarantees conservation of angular momentum. 
In spherical coordinates, the equation of motion becomes

$$
\begin{gathered}
m \frac{d v_{r}}{d t}=F_{r}+\frac{m\left(v_{\theta}^{2}+v_{\phi}^{2}\right)}{r}, \\
m \frac{d\left(r v_{\theta}\right)}{d t}=r F_{\theta}+m r^{2} \sin \theta \cos \theta\left(\frac{d \phi}{d t}\right)^{2}, \text { and } \\
\frac{d\left(m r^{2} \sin ^{2} \theta d \phi / d t\right)}{d t}=r \sin \theta F_{\phi},
\end{gathered}
$$

where $r, \theta$, and $\phi$ are the spherical coordinates and $v_{\phi}=r \sin \theta d \phi / d t$. For the spherical model, $\mathrm{B}=0$ so $F_{\theta}=F_{\phi}=0$ and $F_{r}=q E_{r}$. If $d \phi / d t=0$ initially, then it remains zero. The motion is then in a plane $\phi=$ constant through the polar axis, in which $r$ and $\theta$ play the role of plane polar coordinates. The angular momentum $m r v_{\theta}$ is then constant, and Eq. (7) reduces to the cylindrical case, Eq. (5).

\section{POTENTIAL EQUATION}

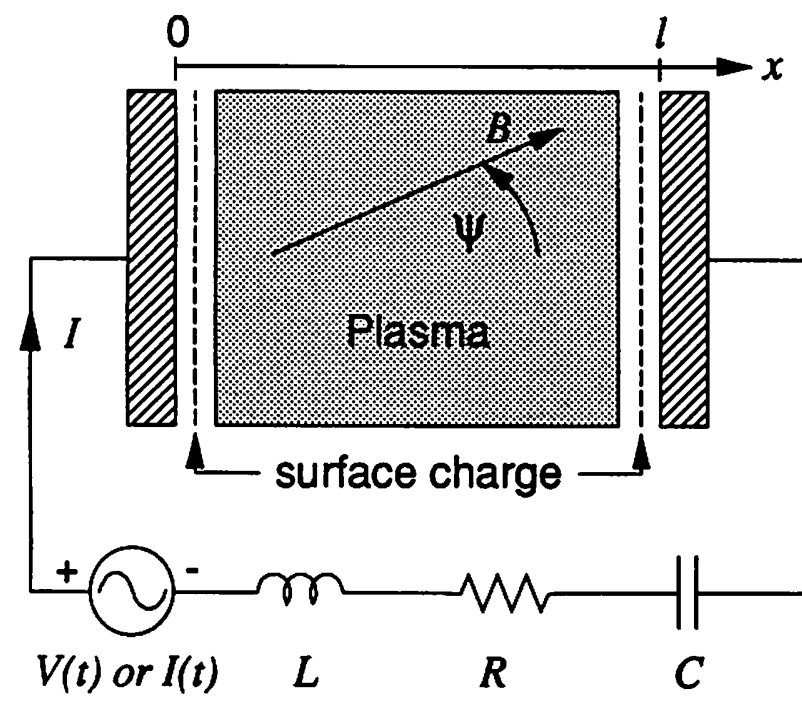

Figure 3. Configuration in planar coordinates with series RLC circuit and voltage/current source. In these coordinates, the particles are charge sheets with motion in the $x$-direction.

The planar, cyclindrical, and spherical configurations for one-dimensional bounded plasma systems are shown in Figures 3 and 4. The current in the external circuit interacts with the plasma current via surface charge on the electrodes. Similarly, the potential within the plasma region is affected by the distribution and motion of space charge, the electrode surface charge, and the current in the external circuit. Thus, we seek a simultaneous solution for the potential and circuit equations. 


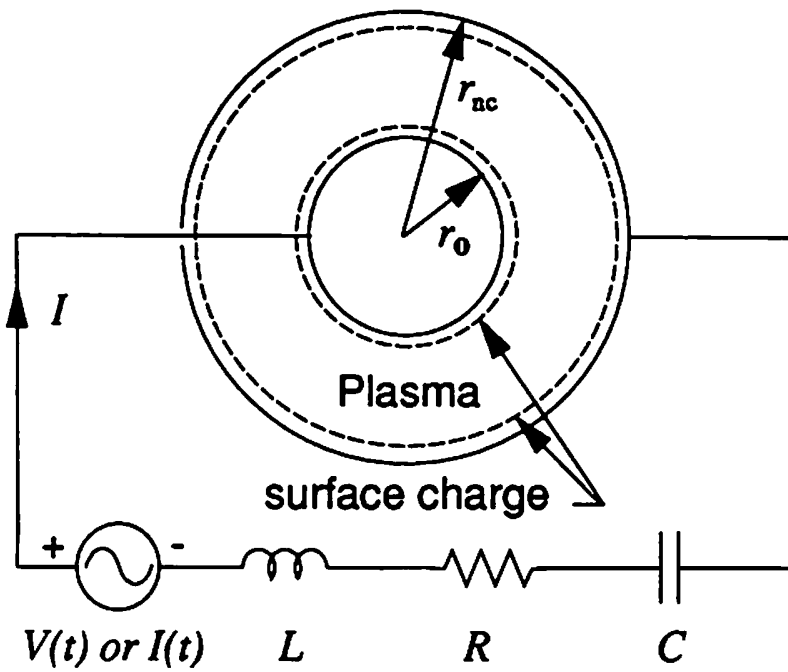

Figure 4. Configuration in cylindrical and spherical coordinates with series RLC circuit and voltage/current source. Particles are infinite annuli with motion in the radial direction in cylindrical coordinates, and spherical shells with radial motion in spherical coordinates.

The boundary conditions for the potential equation are obtained by applying Gauss' Law to the system,

$$
\oint_{S} \mathbf{E} \cdot d \mathbf{S}=\int_{V} \frac{\rho}{\varepsilon} d V+\frac{A_{+} \sigma_{+}+A_{-} \sigma_{-}}{\varepsilon}=0
$$

where the surface $S$ encloses the plasma and electrodes. $A_{+}$refers to the surface area of the positively biased (left/inner) electrode, $A$. to the negatively biased (right/outer) electrode, and $\sigma$ is the surface charge on the respective electrode. Note that $\rho$ has units of charge/volume and $\sigma$ has units of charge/area. Equation (8) is a statement of Gauss' Law; the first part reflects the assumption of an ideal conductor connecting the electrodes to the external circuit elements, the second part expresses conservation of charge in the system.

Applying Gauss' Law about each node of the gridded system, and using the definition of potential, we obtain

planar

$$
\frac{\Phi_{j+1}-2 \Phi_{j}+\Phi_{j-1}}{\Delta x^{2}}=-\frac{\rho_{j}}{\varepsilon}
$$

cylindrical

$$
r_{j+1 / 2} \Phi_{j+1}-2 r_{j} \Phi_{j}+r_{j-1 / 2} \Phi_{j-1}=-\frac{r_{j} \Delta r^{2}}{\varepsilon} \rho_{j} \text {, and }
$$

spherical $\quad r_{j+1 / 2}^{2} \Phi_{j+1}-\left(r_{j+1 / 2}^{2}+r_{j-1 / 2}^{2}\right) \Phi_{j}+r_{j-1 / 2}^{2} \Phi_{j-1}=-\frac{\left(r_{j+1 / 2}^{3}-r_{j-1 / 2}^{3}\right) \Delta r}{3 \varepsilon} \rho_{j}$ 
In the cylindrical and spherical forms, $r_{j \pm 1 / 2}=r_{j} \pm \Delta r / 2$. For all Eq. $(9), j=1,2, \ldots, n c-1$, where $n c$ is the number of cells in the gridded space. These results are equivalent to the flux-conserving method of Birdsall and Langdon [2]. The planar and cylindrical result of Eq. (9) can also be obtained by applying a central difference to the Poisson equation; the finite difference result is different in spherical coordinates.

For a one dimensional system, the boundary conditions can be written

$$
\begin{gathered}
\Phi_{n c}=0 \text { and } \\
E_{0}=\frac{\sigma_{+}}{\varepsilon} .
\end{gathered}
$$

Equation (10) fixes a reference potential for the system without implying a grounded electrode. For the cylindrical and spherical models the inner electrode is driven; the outer electrode serves as the reference potential for the system even when the inner electrode is not present. Equation (11) can be written at one half grid cell from the boundary in conjunction with a central difference applied to the definition of potential to obtain

planar

$$
E_{1 / 2}=\frac{\Phi_{0}-\Phi_{1}}{\Delta x}=\frac{1}{\varepsilon}\left(\sigma_{+}+\rho_{0} \frac{\Delta x}{2}\right),
$$

cylindrical

$$
E_{1 / 2}=\frac{\Phi_{0}-\Phi_{1}}{\Delta r}=\frac{1}{\varepsilon}\left(\sigma_{+} \frac{r_{0}}{r_{1 / 2}}+\frac{\rho_{0}}{2}\left(r_{1 / 2}-\frac{r_{0}^{2}}{r_{1 / 2}}\right)\right) \text {, and }
$$

spherical

$$
E_{1 / 2}=\frac{\Phi_{0}-\Phi_{1}}{\Delta r}=\frac{1}{\varepsilon}\left(\sigma_{+} \frac{r_{0}^{2}}{r_{1 / 2}^{2}}+\frac{\rho_{0}}{3}\left(r_{1 / 2}-\frac{r_{0}^{3}}{r_{1 / 2}^{2}}\right)\right) \text {. }
$$

Equation (9) and its boundary conditions for the gridded system are written in a general matrix form,

$$
\left(\begin{array}{cccccccc}
b_{0} & c_{0} & 0 & \cdot & \cdot & \cdot & & \\
a_{1} & b_{1} & c_{1} & 0 & & & & \\
0 & a_{2} & b_{2} & c_{2} & 0 & & & \\
\cdot & & \cdot & \cdot & \cdot & & & \\
\cdot & & & \cdot & \cdot & \cdot & & \\
\cdot & & & & \cdot & \cdot & \cdot & \\
& & & & & a_{n c-2} & b_{n c-2} & c_{n c-2} \\
& & & & & & a_{n c-1} & b_{n c-1}
\end{array}\right)\left(\begin{array}{c}
\Phi_{0} \\
\Phi_{1} \\
\Phi_{2} \\
\cdot \\
\cdot \\
\cdot \\
\Phi_{n c-2} \\
\Phi_{n c-1}
\end{array}\right)^{t}=f\left(\begin{array}{c}
d_{0} \\
d_{1} \\
d_{2} \\
\cdot \\
\cdot \\
\cdot \\
d_{n c-2} \\
d_{n c-1}
\end{array}\right)^{t}
$$


The superscript indicates the quantity is evaluated at time $t$. The matrix elements in planar coordinates are

$$
\begin{array}{lll}
b_{0}=-1, & a_{j}=1, & j=1,2, \ldots, n c-1 ; \\
& b_{j}=-2, & j=1,2, \ldots, n c-1 ; \\
c_{j}=1, & j=0,1, \ldots, n c-2 ; \\
d_{0}=\frac{\sigma_{+}}{\Delta x}+\frac{\rho_{0}^{t}}{2}, & d_{j}=\rho_{j}^{t}, & j=1,2, \ldots, n c-1 ;
\end{array}
$$

and

$$
f=-\frac{\Delta x^{2}}{\varepsilon}
$$

The matrix elements in cylindrical coordinates are

$$
\begin{aligned}
& a_{j}=r_{j-1 / 2}, \quad j=1,2, \ldots, n c-1 ; \\
& b_{0}=-r_{1 / 2}, \quad b_{j}=-2 r_{j}, \quad j=1,2, \ldots, n c-1 \text {; } \\
& c_{j}=r_{j+1 / 2}, \quad j=0,1, \ldots, n c-2 \text {; } \\
& d_{0}=\sigma_{+} \frac{r_{0}}{\Delta r}+\frac{\rho_{0}^{t}}{2 \Delta r}\left(r_{1 / 2}^{2}-r_{0}^{2}\right), \quad d_{j}=r_{j} \rho_{j}^{t}, \quad j=1,2, \ldots, n c-1 ; \\
& \text { and } \quad f=-\frac{\Delta r^{2}}{\varepsilon} \text {. }
\end{aligned}
$$

The matrix elements in spherical coordinates are

$$
\begin{array}{lll}
a_{j}=r_{j-1 / 2}^{2}, & j=1,2, \ldots, n c-1 ; \\
b_{0}=-r_{1 / 2}^{2}, & b_{j}=-\left(r_{j-1 / 2}^{2}+r_{j+1 / 2}^{2}\right), & j=1,2, \ldots, n c-1 ; \\
c_{j}=r_{j+1 / 2}^{2}, & j=0,1, \ldots, n c-2 ; \\
d_{0}=3 \sigma_{+}^{2} r_{0}^{2}+\rho_{0}^{i}\left(r_{1 / 2}^{3}-r_{0}^{3}\right), & d_{j}=\left(r_{j+1 / 2}^{3}-r_{j-1 / 2}^{3}\right) \rho_{j}^{t}, & j=1,2, \ldots, n c-1 ; \\
& f=-\frac{\Delta r}{3 \varepsilon} . &
\end{array}
$$

and

When the center conductor is not present in the curvilinear models, the boundary conditions must be modified. From Gauss' Law, the electric field at the origin must be zero. Integrating Gauss' Law from the origin to $r=r_{1 / 2}$, we obtain the modified form of Eq. (12) for the hollow cylindrical system [2, Section $14-10]$, 


$$
E_{1 / 2}=\frac{\Phi_{0}-\Phi_{1}}{\Delta r}=\frac{1}{\varepsilon}\left(\frac{\rho_{0}}{2} r_{1 / 2}\right)
$$

The coefficients are still given by Eq. (15), with the modification that

$$
d_{0}=\frac{\rho_{0}^{t}}{2 \Delta r} r_{1 / 2}^{2}
$$

The modification of Eq. (16) for removal of the center electrode in the spherical system is similarly

$$
d_{0}=\rho_{0}^{t} r_{1 / 2}^{3}
$$

\section{CIRCUIT}

The external circuit is coupled to Eqs. (13)-(16) through conservation of charge at each wall,

$$
A \Delta \sigma=Q_{\text {conv }}+\Delta Q \text {, }
$$

where $Q_{\text {conv }}$ is the charge deposited by the convection (particle) current and $\Delta Q$ is the charge deposited by the external circuit current, both over some interval in time. Equation (20) is applied at the positively biased electrode as shown in Figures 3 and 4, guaranteeing conservation of charge at all times. The same logic can also be applied to the other electrode; however, the surface charge on the second electrode is determined readily from Eq. (8) when the first surface charge is known. The charge conservation equation becomes

$$
\sigma=\sigma^{s-\Delta t}+\frac{Q_{\text {conv }}^{t}+Q^{t}-Q^{t-\Delta t}}{A},
$$

where $Q$ is the charge on one plate of the external circuit capacitor. An alternate method of coupling the circuit to the potential matrix is applying continuity of current (Kirchhoff's Current Law) at the boundary [2, Section 16-9],

$$
\frac{\partial \sigma}{\partial t}=J_{c o n v}+\frac{I}{A}
$$

where $J_{\text {conv }}$ is the plasma convection current at the electrode. The methods are equivalent when a first-order backward difference is used for $\partial \sigma / \partial t$ and $I=\partial Q / \partial t$. Since $Q_{\text {conv }}$ is in general a noisy quantity in a particle simulation, any other quantity in Eqs. (20) and (22) will contain similar noise. Thus Eq. (20) causes the wall charge $\sigma$ to be noisy as might be expected, because the capacitor charge reacts to the particle convection current only through the wall charge; i.e., particles absorbed 
by the wall contribute immediately to $\sigma$, but the charge drains slowly to the capacitor through currents. It can be shown that Eq. (22) results in the convection current being absorbed gradually into $\sigma$, so the noise is induced in the capacitor charge $Q$ (and consequently in the external current I) to satisfy conservation of charge. Therefore we use the conservation of charge method of Eq. (20).

\section{GENERAL SERIES RLC CIRCUIT}

Four cases cover the full range of external circuit parameters. For the general voltage-driven series RLC circuit, the capacitor charge $Q$ is advanced using Kirchhoff's Voltage Law,

$$
L \frac{d^{2} Q}{d t^{2}}+R \frac{d Q}{d t}+\frac{Q}{C}=V(t)+\Phi_{n c}-\Phi_{0} .
$$

The polarity of the source and resultant positive current are shown in Figures 3 and 4 . The general circuit equation is finite differenced using the second-order backward Euler representation of the first derivative,

$$
\left(\frac{d Q}{d t}\right)^{t}=\frac{3 Q^{t}-4 Q^{t-\Delta t}+Q^{t-2 \Delta}}{2 \Delta t}
$$

and the second derivative,

$$
\begin{aligned}
\left(\frac{d^{2} Q}{d t^{2}}\right)^{t} & =\frac{3(d Q / d t)^{t}-4(d Q / d t)^{t-\Delta t}+(d Q / d t)^{t-2 \Delta}}{2 \Delta t} \\
& =\frac{9 Q^{t}-24 Q^{t-\Delta t}+22 Q^{t-2 \Delta}-8 Q^{t-3 \Delta t}+Q^{t-4 \Delta t}}{4 \Delta t^{2}} .
\end{aligned}
$$

The latter is obtained by a second application of the first derivative to $Q$. An alternate 4 point difference for the second derivative is given by:

$$
\left(\frac{d^{2} Q}{d t^{2}}\right)^{t}=\frac{2 Q^{t}-5 Q^{t-\Delta t}+4 Q^{t-2 \Delta t}-Q^{t-3 \Delta t}}{\Delta t^{2}} .
$$

The charge on the capacitor is not known at $t$. Combining Eqs. (23)-(25), we obtain

$$
Q^{t}=\frac{V(t)+\Phi_{n c}^{t}-\Phi_{0}^{t}-K^{t}}{\alpha_{0}},
$$


where

$$
\begin{gathered}
K^{t}=\alpha_{1} Q^{t-\Delta t}+\alpha_{2} Q^{t-2 \Delta t}+\alpha_{3} Q^{t-3 \Delta t}+\alpha_{4} Q^{t-4 \Delta t}, \\
\alpha_{0}=\frac{9}{4} \frac{L}{\Delta t^{2}}+\frac{3}{2} \frac{R}{\Delta t}+\frac{1}{C}, \\
\alpha_{1}=-6 \frac{L}{\Delta t^{2}}-2 \frac{R}{\Delta t}, \\
\alpha_{2}=\frac{11}{2} \frac{L}{\Delta t^{2}}+\frac{1}{2} \frac{R}{\Delta t}, \\
\alpha_{3}=-2 \frac{L}{\Delta t^{2}}, \text { and } \\
\alpha_{4}=\frac{1}{4} \frac{L}{\Delta t^{2}} .
\end{gathered}
$$

Combining the potential equation results, Eqs. (13)-(16), with the circuit equation results, Eqs. (27) and (28), using the boundary condition, Eq. (21), we obtain the self consistent field solution matrix for the voltage-driven series RLC circuit case. The matrix can still be represented by Eq. (13), replacing elements of Eqs. (14)-(16) as follows. In the planar model,

$$
\begin{gathered}
b_{0}=-1-\frac{\Delta x}{\alpha_{0} \varepsilon A} \text { and } \\
d_{0}=\frac{\rho_{0}^{t}}{2}+\frac{\sigma_{+}^{t-\Delta t}}{\Delta x}+\frac{1}{A \Delta x}\left(Q_{\text {conv }}^{t}-Q^{t-\Delta t}+\frac{V(t)-K}{\alpha_{0}}\right) .
\end{gathered}
$$

In the cylindrical model,

$$
\begin{gathered}
b_{0}=-r_{1 / 2}-\frac{\Delta r}{2 \pi \varepsilon \alpha_{0} h} \text { and } \\
d_{0}=\frac{r_{1 / 2}^{2}-r_{0}^{2}}{2 \Delta r} \rho_{0}^{t}+\frac{r_{0}}{\Delta r} \sigma_{+}^{t-\Delta t}+\frac{1}{2 \pi h \Delta r}\left(Q_{c o n v}^{t}-Q^{t-\Delta t}+\frac{V(t)-K}{\alpha_{0}}\right) .
\end{gathered}
$$

In the spherical model,

$$
\begin{gathered}
b_{0}=-r_{1 / 2}^{2}-\frac{\Delta r}{4 \pi \varepsilon \alpha_{0}} \text { and } \\
d_{0}=\left(r_{1 / 2}^{3}-r_{0}^{3}\right) p_{0}^{t}+3 r_{0}^{2} \sigma_{+}^{t-\Delta t}+\frac{3}{4 \pi}\left(Q_{c o n v}^{t}-Q^{t-\Delta t}+\frac{V(t)-K}{\alpha_{0}}\right) .
\end{gathered}
$$


Here, $A$ is area of the planar electrodes and $h$ is axial length of the cylindrical system. The solution is then self-consistent and second-order accurate for the general circuit case. The matrix can be solved using any algorithm optimized for tridiagonal matrices [3].

\section{OPEN CIRCUIT (FLOATING OUTER ELECTRODE)}

When $C \rightarrow 0$, the impedance of the external circuit approaches infinity, becoming an open circuit. The potentials on the boundaries are floating; no circuit solution is required since there is no external current. The surface charges on the electrodes influence the potential as always, but the electrodes cannot exchange charge via external current. In this case, the field solution is given by Eqs. (13)-(16), with

$$
\sigma_{+}^{\prime}=\sigma_{+}^{s-\Delta s}+Q_{\text {conv }}^{l} .
$$

\section{SHORT-CIRCUIT}

When $R=L=0$ and $C \rightarrow \infty$, the external circuit is a short, with

$$
\Phi_{0}-\Phi_{n c}=V(t) .
$$

The short-circuit case is applied in practice when

planar

$$
\frac{C}{\varepsilon A / l}>10^{5},
$$

cylindrical

$$
\frac{C}{2 \pi \varepsilon h / \ln \left(r_{n c} / r_{0}\right)}>10^{5},
$$

spherical

$$
\frac{C}{4 \pi \varepsilon r_{n c} r_{d}\left(r_{n c}-r_{0}\right)}>10^{5},
$$

where $l$ is the length of the planar plasma region.

The field solution is still given by Eqs. (13)-(16), with

$$
a_{1}=b_{0}=c_{0}=d_{0}=0,
$$

and 
planar

$$
d_{1}=\rho_{1}^{t}-\frac{V(t)}{f},
$$

cylindrical

$$
d_{1}=r_{1} \rho_{1}^{t}-\frac{r_{1 / 2} V(t)}{f} \text {, and }
$$

spherical

$$
d_{1}=\left(r_{3 / 2}^{3}-r_{1 / 2}^{3}\right) \rho_{1}^{t}-\frac{r_{1 / 2}^{2} V(t)}{f}
$$

Equation (35) eliminates the first row of Eq. (13). In Eq. (36), $f$ depends on the model as given in Eqs. (14)-(16). Note that the wall charge is no longer required to solve the potential equation. Wall charge is found using Eq. (12) once the potentials have been determined, and the current is found by finite differencing Eq. (22),

$$
I^{t-\Delta / 2}=A\left(J_{\text {conv }}+\frac{\sigma^{t}-\sigma^{t-\Delta t}}{\Delta t}\right) .
$$

Determining the current in this way produces a noisy result as discussed above; however, with a short between electrodes, we expect large currents with rapid changes since potential differences cannot exist along an ideal conductor. Note that here $I$ is only a diagnostic quantity, so the time-centering is not a problem.

\section{CURRENT-DRIVEN CIRCUIT}

The final case is the current-driven external circuit. An ideal current source is assumed which can drive the specified time-varying current $I(t)$. The external circuit elements $R, L$, and $C$ are ignored since an ideal current source is an open circuit. Then Eqs. (13)-(16) are applied with the wall charge found by finite differencing Eq. (22) for diagnostic purposes.

\section{INITIAL CONDITIONS}

The multi-point finite difference methods require initial values for the $Q^{n}$, where $n \leq 0$. Physically, these values are used to obtain the desired initial conditions for the circuit equation, Eq. (23). For example, the initial charge on the capacitor, $Q^{0}$, and the initial current in the external circuit, $I^{0}$, form a complete set of initial conditions for the differential equation. However, the finite difference requires five initial values for $Q$ (four for the 4-point method). There are several ways the conditions can be obtained.

The traditional method for starting a multi-point scheme (second or higher order accurate) is to use a 2-point method (first order accurate) to obtain the required initial values. A smaller timestep is used with the 2-point method to maintain the same accuracy. This presents a problem for a PIC code; the time-centered mover is initialized such that positions are known at integral 
timesteps, while velocities are known at half timesteps [2]. Thus, it is difficult to switch to a new $\Delta t$ and maintain the time centering. Also, switching schemes is inefficient from a coding standpoint. In addition, the stability of the starter method must be considered in relation to the circuit parameters $R, L$, and $C$.

Another method of initializing the solver is to solve the circuit equations analytically. To do this, we must replace the plasma by a known impedance. Using the vacuum capacitance of the plasma region is the obvious choice; physically, this means there is no plasma until $t=0^{+}$. If plasma is then introduced, the impedance changes abruptly and the circuit has been conditioned for a different system. This problem is less severe when the plasma is generated at a slow rate since the impedance change is gradual.

If the method turns out to be stable, the initial conditions will be damped regardless of the value (this includes desired initial conditions as well as error in the initial conditions). If the method is unstable, any error in the initial conditions grows exponentially. If the method is marginally stable, any error in the initial conditions remains in the solution, neither growing nor damping.

\section{STABILITY}

We now explore stability of the circuit equation, Eq. (27). As is customary for stability analysis [4], we neglect the driving terms and study the homogeneous circuit equation

$$
L \frac{d^{2} Q}{d t^{2}}+R \frac{d Q}{d t}+\frac{Q}{C}=0
$$

We study the stability of the 5-point circuit difference, Eqs. (24)-(25), as well as the 4-point difference, Eq. (24) and (26).

In the limit of no inductance, $L \rightarrow 0$, both methods produce

$$
Q^{t}\left(3+\frac{2 \Delta t}{R C}\right)-4 Q^{t-\Delta t}+Q^{t-2 \Delta t}=0 \text {. }
$$

Letting $Q^{s}=Q^{0} e^{r}$ and $\xi=e^{\gamma \Delta s}$ we obtain

$$
Q^{t}=\xi Q^{t-\Delta t}=\xi^{2} Q^{t-2 \Delta t},
$$

where $|\xi| \leq 1$ is required for stability. Here, $\gamma$ and $\xi$ are arbitrary complex variables. Then the characteristic stability equation for Eq. (39) is

$$
\xi^{2}(3+2 \Delta t / R C)-4 \xi+1=0 .
$$


The roots are

$$
\xi=\frac{2 \pm \sqrt{1-2 \Delta t / R C}}{3+2 \Delta t / R C}
$$

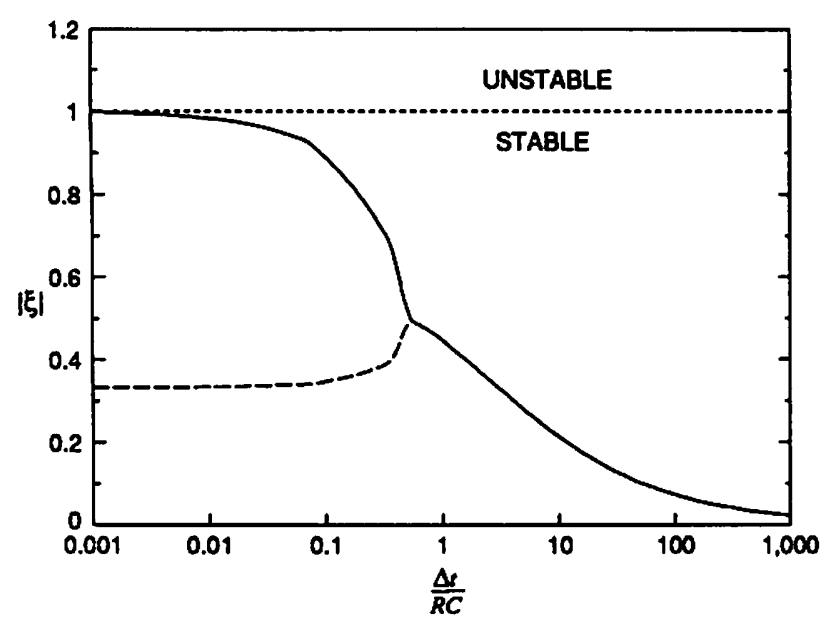

Figure 5. Stability roots in the limit $L \rightarrow 0$. Since $|\xi| \leq 1$ everywhere, the method is stable. The scheme can only follow the RC time when $\Delta t \leq R C / 2$.

As shown in Figure 5, both methods are stable in the limit $L \rightarrow 0$ for all positive, real $\Delta t / R C$.

Now we attack the more difficult general case. The general characteristic stability equations for the four and five point schemes are respectively

$$
\begin{gathered}
\xi^{3}\left(2+\frac{3}{2} \tau_{1}+\tau_{2}^{2}\right)-\xi^{2}\left(5+2 \tau_{1}\right)+\xi\left(4+\frac{1}{2} \tau_{1}\right)-1=0 \text { and } \\
\xi^{4}\left(9+6 \tau_{1}+4 \tau_{2}^{2}\right)-\xi^{3}\left(24+8 \tau_{1}\right)+\xi^{2}\left(22+2 \tau_{1}\right)-8 \xi+1=0,
\end{gathered}
$$

where the normalized times are $\tau_{1}=R \Delta t / L$ and $\tau_{2}=\Delta t / \sqrt{L C}$. We obtain the roots of Eq. 31 using the Lin-Bairstow method [5], which gives the complex roots of polynomials. Figures 6 and 7 show the stability of the four and five point methods, respectively, for a wide range of $\tau_{1}$ and $\tau_{2}$. 


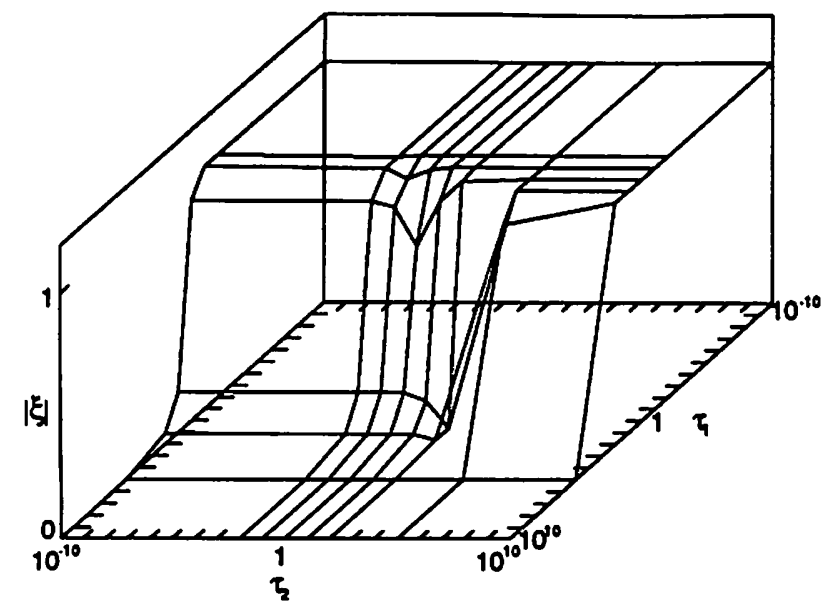

(a)

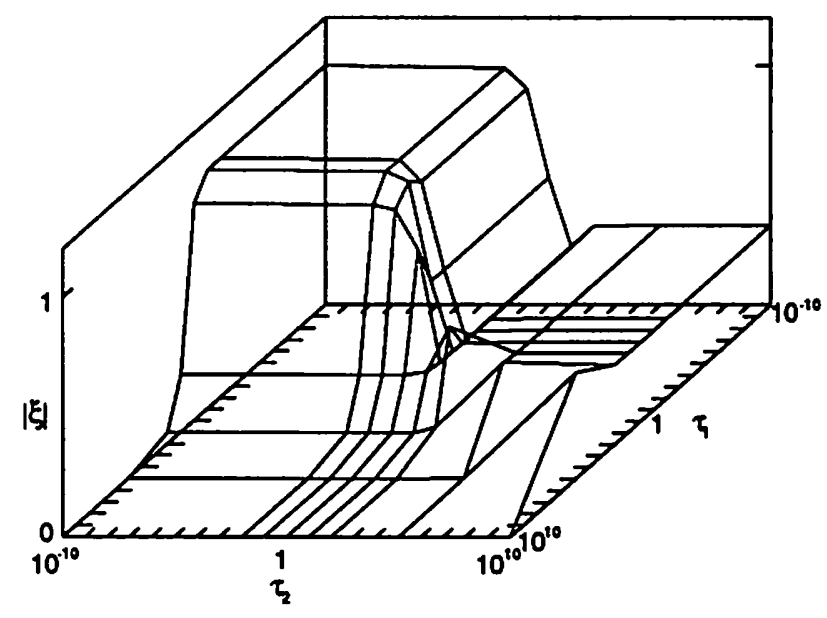

(c)

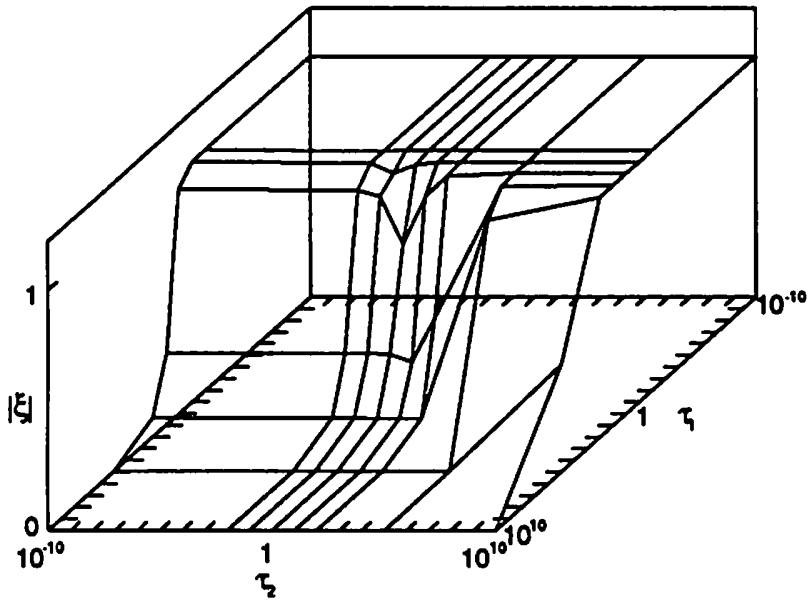

(a)

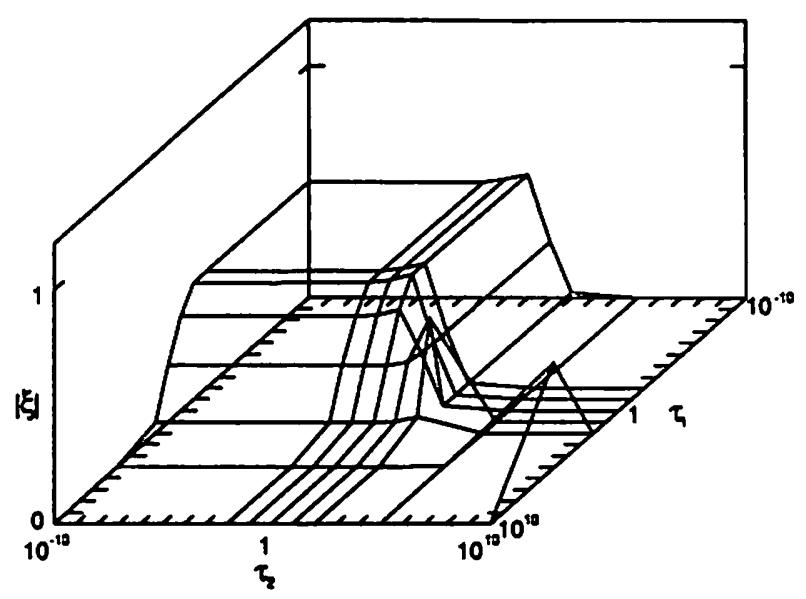

(b)

Figure 6. Magnitude of the three roots of the four point method, whose characteristic equation is Eq. (43). Since for all three roots, $\mid \xi_{1,23} \leqslant 1$, the four point difference method is stable over the range shown.

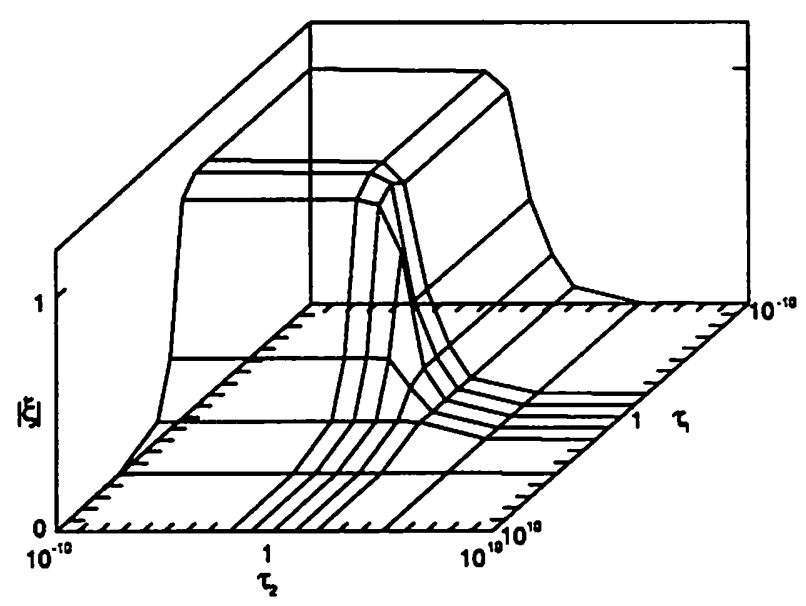

(b) 


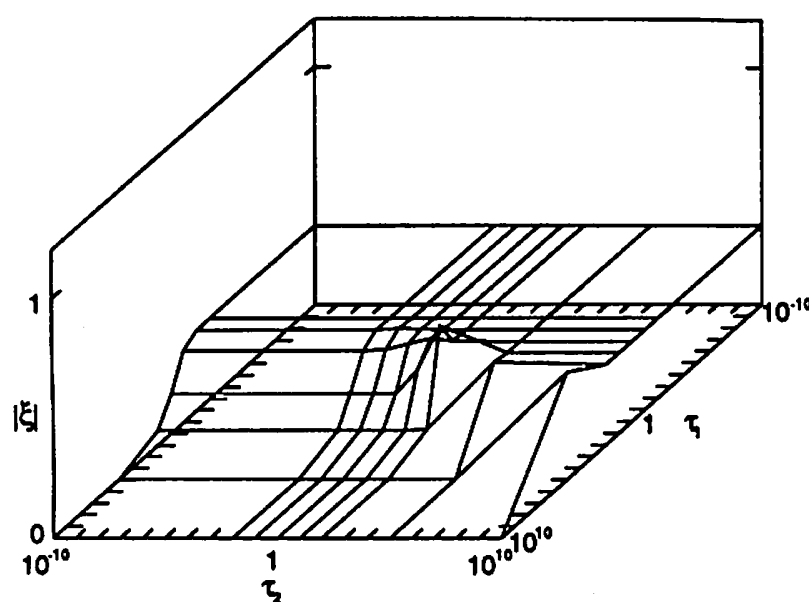

(c)

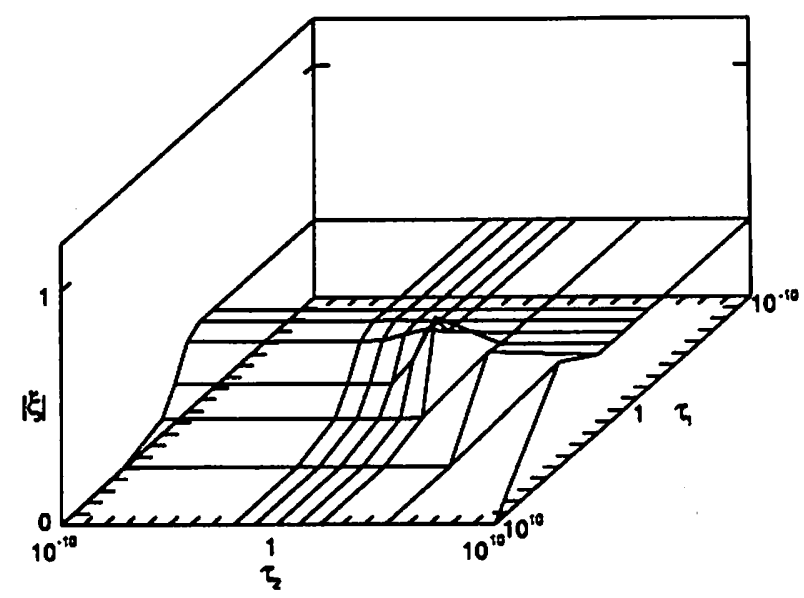

(d)

Figure 7. Magnitude of the four stability roots of the five point method, whose characteristic equation is Eq. (44). Since all four roots, $\left|\xi_{1,2,3,4}\right| \leq 1$, the five point difference scheme is stable over the range shown.

\section{SIMULATION ACCURACY}

As discussed above, the circuit and field solution is second-order accurate. We now demonstrate the accuracy of the five point circuit as implemented in the code, PDP1. To compare the simulation results with analytic circuit theory, the permittivity of the plasma region is taken as $10^{20}$ and no plasma is used. Then we have a passive voltage-driven series RLC circuit. The current for a sinusoidal driving voltage $V=\sin (\omega t+\theta)$ can be predicted using

$$
\begin{aligned}
I & =\frac{a_{2} V /(\omega Z) \cos (\theta-\delta)+V / Z \sin (\theta-\delta)}{a_{2} / a_{1}-1} \exp \left(a_{1} t\right) \\
& +\frac{a_{1} V /(\omega Z) \cos (\theta-\delta)+V / Z \sin (\theta-\delta)}{a_{1} / a_{2}-1} \exp \left(a_{2} t\right)+\frac{V}{Z} \sin (\omega t+\theta-\delta),
\end{aligned}
$$

where $\omega$ is the angular frequency of the voltage, $\theta$ is the initial phase of the voltage, and

$$
\begin{gathered}
Z=\sqrt{R^{2}+\left(\omega L-\frac{1}{\omega C}\right)^{2}}, \\
\delta=\operatorname{asin}\left(\frac{\omega L-1 /(\omega C)}{Z}\right), \\
a_{1}=-\frac{R}{2 L}+\sqrt{\frac{R^{2}}{4 L^{2}}-\frac{1}{L C}}, \text { and }
\end{gathered}
$$




$$
a_{2}=-\frac{R}{2 L}-\sqrt{\frac{R^{2}}{4 L^{2}}-\frac{1}{L C}}
$$

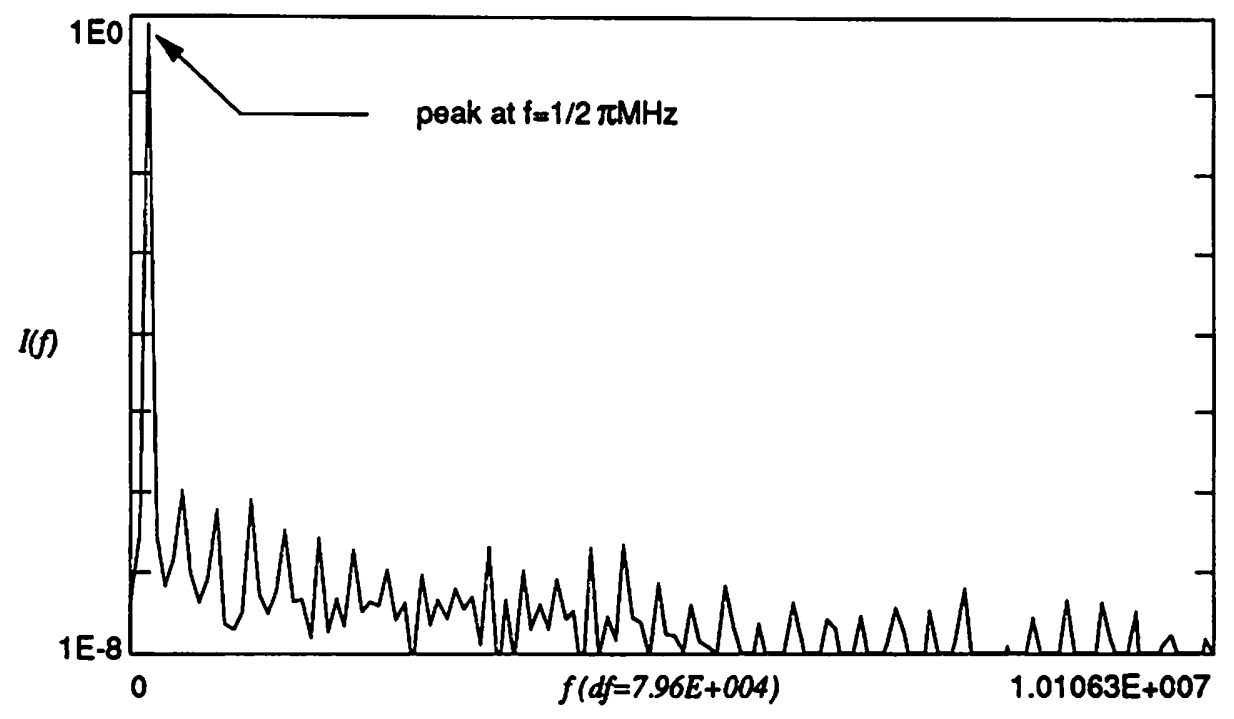

Figure 8. The frequency spectrum of the steady-state current obtained from PDP1.

We choose $R=1, C=5 \times 10^{-6}, L=10^{-6}$, and $\omega=10^{6}$, and initial conditions $Q(0)=0$ and $I(0)=0$. The code PDP1, using the same parameters and timestep $\Delta t_{0}=2 \pi / 128 \omega$, gives the results shown in Figures 8 and 9. Here, $\tau_{1}=0.049$ and $\tau_{2}=0.022$ for the baseline case. Note that the $\tau$ scale with $\Delta t / \Delta t_{0}$.

From the frequency spectrum of the current shown in Figure 8, we see the driven frequency peak is six orders of magnitude larger than the magnitude at other frequencies, indicating nearly a pure sinusoid. Since PDP1 stores results in single precision (32 bits), we expect roundoff error in the sixth or seventh significant digit, so the powers less than $10^{-7}$ are neglible. Comparing the phases $I(t)$, we see the the PDP1 results follow Eq. (45) closely, with increasing phase error as $\Delta t$ increases. From the history of the current, we see the initial transient due to the charging of the capacitor from $Q(0)=0$. 


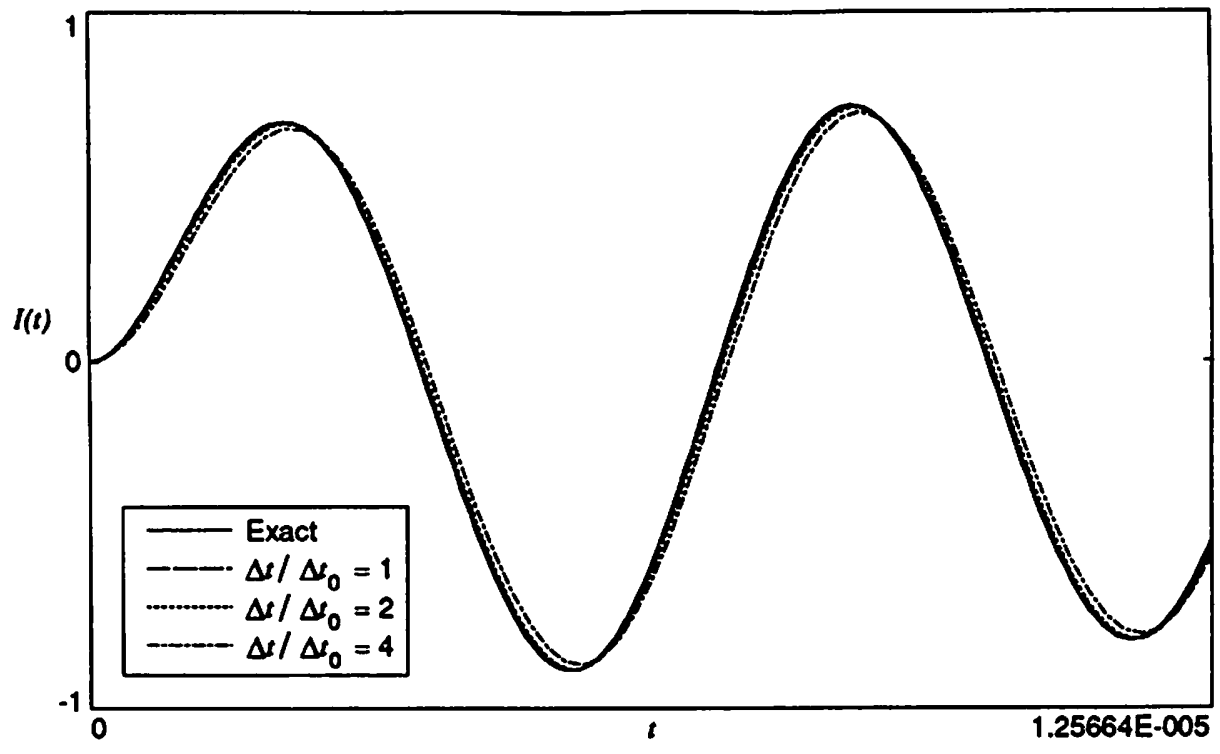

Figure 9. PDP1 output for voltage-driven series RLC circuit. The exact current predicted by Eq. (45) compared to the results of the PDP1 circuit solver at various ratios of $\Delta t / \Delta t_{0}$ where $\Delta t_{0}=2 \pi /(128 \omega)$. Note the transient charging of the external capacitor.

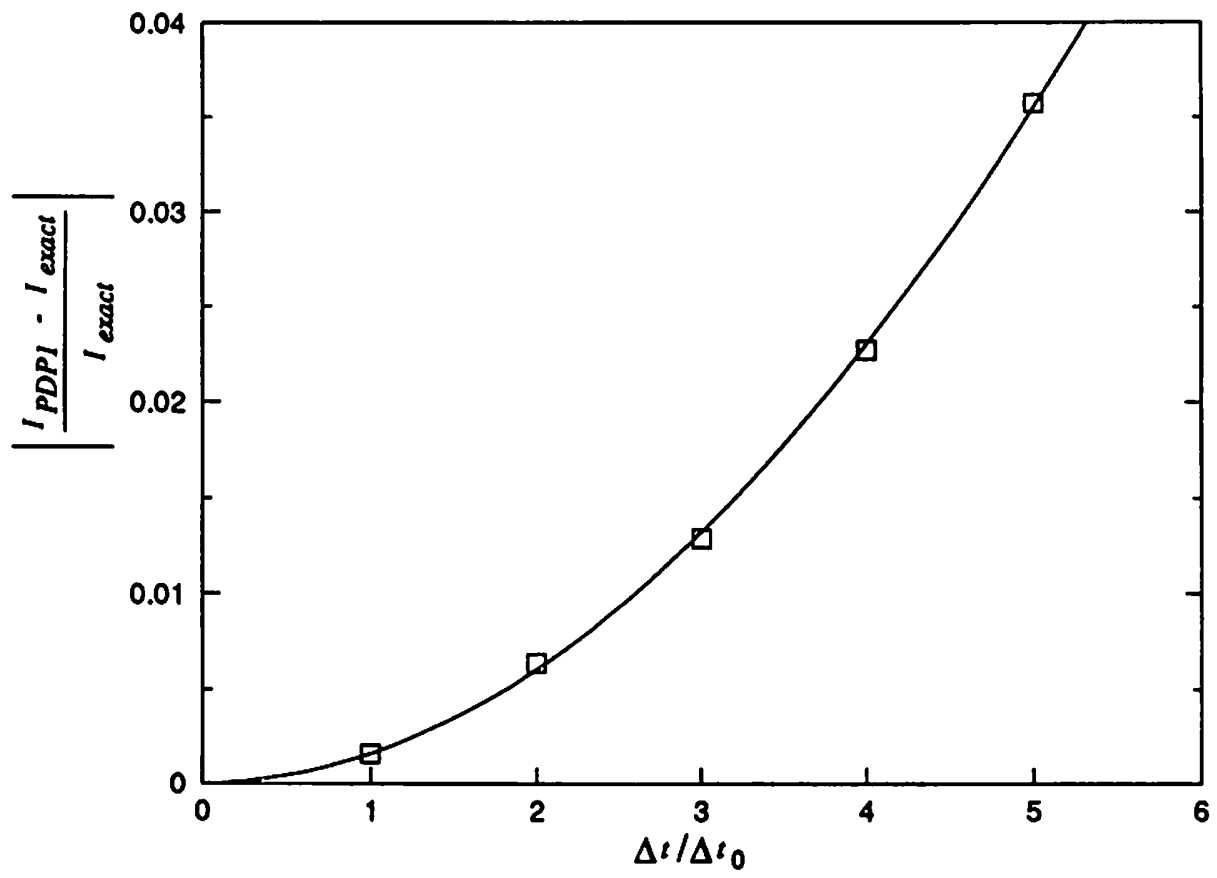

Figure 10. The relative error, $\mid\left(I_{\text {exed }}-I_{P D P_{1}}\right) / I_{\text {exed }} l$, versus $\Delta t / \Delta I_{0}$ compared with a power fit. The exponent of the best power fit is 1.94 . 
The relative error, plotted in Figure 10, follows the curve $0.00158(\Delta t)^{1.94}$ closely. An ideal second order accurate scheme would result in a power fit exponent of 2 . This demonstrates second order accuracy, with errors resulting from truncation of the finite difference at $\Delta t^{2}$ terms.

\section{CONCLUSION}

A method for the simultaneous solution of the coupled potential and external circuit equations for one-dimensional electrostatic plasma particle simulations is presented. The method is stable over many orders of magnitude for the values of the RLC circuit elements, and can in principle be extended to arbitrary external circuits.

The method is implemented in the codes PDP1 (Plasma Device Planar 1 Dimension), PDC1 (Cylindrical), and PDS1 (Spherical) $^{\dagger}$. These codes have been used to simulate many complete bounded plasma devices [6-11], including voltage-driven RF discharges, plasma immersion ion implantation devices, and Q-machines. The codes have performed reliably, generating many interesting discussions and discoveries.

\section{ACKNOWLEDGMENTS}

We are grateful to C. K. Birdsall for his comments on the manuscript and many useful discussions on PIC fundamentals as well as assistance verifying the model with known results. We also thank W. S. Lawson allowing us to borrow liberally from his code PDW1, and E. Horowitz for his insight regarding particle weighting in curvilinear geometry.

This work supported in part by DOE contract DE-FG03-86ER53220 and ONR contract N00014-85-K-0809.

†Codes available through Software Distribution Office, Industrial Liason Program, Cory Hall, University of California, Berkeley, CA 94720. 


\section{REFERENCES}

1. W. S. Lawson, "Particle Simulation of Bounded 1D Plasma Systems," J. Comp. Phys., 80, 253-276 (1989).

2. C. K. Birdsall and A. B. Langdon, Plasma Physics Via Computer Simulation, McGraw-Hill (1985).

3. W. H. Press, B. P. Flannery, S. A. Teukolsky, and W. Y. Vetterling, Numerical Recipes in C, Cambridge University Press (1988).

4. C. W. Gear, Numerical Initial Value Problems in Ordinary Differential Equations, Prentice-Hall (1971).

5. R. W. Hornbeck, Numerical Methods, Prentice-Hall (1975).

6. M. V. Alves, V. Vahedi, and C. K. Birdsall, Bull. APS, 34:2028 (1989).

7. I. J. Morey, V. Vahedi, and J. P. Verboncoeur, Bull. APS, 34:2028 (1989).

8. I. J. Morey, V. Vahedi, and J. P. Verbonceour, Proceedings of the $13^{\text {th }}$ Conference on the Numerical Simulation of Plasmas, PMB 11 (1989).

9. M. V. Alves, V. Vahedi, and C. K. Birdsall, Proceedings of the $13^{\text {th }}$ Conference on the Numerical Simulation of Plasmas, PW11 (1989).

10. M. V. Alves, V. Vahedi, and C. K. Birdsall, Proceedings of the $42^{\text {nd }}$ Annual Gaseous Electronics Conference, 61 (1989).

11. I. J. Morey, V. Vahedi, J. P. Verboncoeur, and M. A. Lieberman, Proceedings of the $42^{\text {nd }}$ Annual Gaseous Electronics Conference, 61 (1989). 